Helsinki University of Technology Institute of Mathematics Research Reports

\title{
ANALYSIS OF FINITE ELEMENT METHODS FOR THE BRINKMAN PROBLEM
}

\author{
Mika Juntunen Rolf Stenberg
}



Helsinki University of Technology Institute of Mathematics Research Reports

\title{
ANALYSIS OF FINITE ELEMENT METHODS FOR THE BRINKMAN PROBLEM
}

\author{
Mika Juntunen Rolf Stenberg
}


Mika Juntunen, Rolf Stenberg: Analysis of finite element methods for the Brinkman problem; Helsinki University of Technology Institute of Mathematics Research Reports A557 (2009).

\begin{abstract}
The parameter dependent Brinkman problem, covering a field of problems from the Darcy equations to the Stokes problem, is studied. A mathematical framework is introduced for analyzing the problem. Using this we prove uniform a priori and a posteriori estimates for two families of finite element methods. We also discuss Nitshe's method for imposing boundary conditions.
\end{abstract}

\title{
AMS subject classifications: $65 \mathrm{~N} 30$
}

Keywords: Brinkman equation, Stokes equation, Darcy equation, Nitsche's method, mixed finite element methods, stabilized methods

\section{Correspondence}

Helsinki University of Technology

Department of Mathematics and Systems Analysis

P.O. Box 1100

FI-02015 TKK

Finland

mika.juntunen@tkk.fi,rolf.stenberg@tkk.fi

ISBN 978-951-22-9604-0 (print)

ISBN 978-951-22-9605-7 (PDF)

ISSN 0784-3143 (print)

ISSN 1797-5867 (PDF)

Helsinki University of Technology

Faculty of Information and Natural Sciences

Department of Mathematics and Systems Analysis

P.O. Box 1100, FI-02015 TKK, Finland

email: math@tkk.fi http://math.tkk.fi/ 


\section{Introduction}

The purpose of this paper is to analyze finite element methods for the Brinkman equations modeling porous media flow. The model is usually derived by homogenization assuming a high porosity, cf. [11, 1, 2, 3, 15]. The equations are, in fact, a whole range of equations with Darcy's equations and Stokes equations as limits. As a consequence, it is not trivial to design efficient finite element methods. If they are efficient for the Darcy problem that is not necessarily the case for Stokes, and vice versa. Tied to this are the norms used in the analysis for the velocity and pressure, respectively. Roughly speaking, they change place when going from one extreme to the other.

The plan of the paper is as follows. In the next section we introduce a framework using two scales of norms for analyzing the problem. We do not use the approach of [12] since that does not include the Stokes limit. In Section 3 we consider a family of classical mixed finite element methods. We prove the stability (in the chosen norms) and derive both a priori and a posteriori error estimates. Next, we perform the same analysis for a family of stabilized finte element methods. In Section 5 we follow [8] and discuss the enforcement of Dirichlet boundary conditions by Nitsche's method.

In a forthcoming paper [9] we present the results of numerical tests with the finite element methods.

\section{The Brinkman problem}

Let $\Omega \subset \mathbb{R}^{N}$ be a domain with polygonal or polyhedral boundary. The Brinkman problem is the parameter dependent equations

$$
\begin{aligned}
-t^{2} \boldsymbol{A} \boldsymbol{u}+\boldsymbol{u}+\nabla p & =\boldsymbol{f} \text { in } \Omega, \\
\operatorname{div} \boldsymbol{u} & =g \text { in } \Omega,
\end{aligned}
$$

where the parameter $0 \leq t \leq C$. Above we denote $\boldsymbol{A}=\operatorname{div} \boldsymbol{\varepsilon}(\boldsymbol{u})$ and $\varepsilon(\boldsymbol{u})=\left(\nabla \boldsymbol{u}+\nabla \boldsymbol{u}^{T}\right) / 2$. For $t>0$ the equations are formally a Stokes problem for which we assume homogeneous essential boundary conditions

$$
\boldsymbol{u}=\mathbf{0} \text { on } \partial \Omega \text {. }
$$

In the limit $t=0$, we obtain the Darcy problem with the natural boundary conditions

$$
\boldsymbol{u} \cdot \boldsymbol{n}=0 \text { on } \partial \Omega .
$$

Since the boundary conditions are homogenous, the compatibility condition $g \in L_{0}^{2}(\Omega)$ is required for the load in both cases. The same condition; $p \in$ $L_{0}^{2}(\Omega)$, is imposed in order to have a unique pressure.

The natural energy norm for the velocity is

$$
\|\boldsymbol{v}\|_{t}^{2}=t^{2}\|\varepsilon(\boldsymbol{v})\|_{0}^{2}+\|\boldsymbol{v}\|_{0}^{2}
$$


and the natural solution space is $\boldsymbol{V}$; the completion of $\left[C_{0}^{\infty}(\Omega)\right]^{N}$ with respect to this norm. For $t>0$ we have

$$
\boldsymbol{V}=\left[H_{0}^{1}(\Omega)\right]^{N}
$$

but the equivalence is not uniform, for $0<t \leq C$ it holds

$$
C_{1} t\|\boldsymbol{v}\|_{1} \leq\|\boldsymbol{v}\|_{t} \leq C_{2}\|\boldsymbol{v}\|_{1} .
$$

(Here and in the sequel all constants $C$ and $C_{i}$ are assumed independent of $t$ and the mesh parameter $h$.) For $t=0$ the space is

$$
\boldsymbol{V}=\left[L^{2}(\Omega)\right]^{N} .
$$

Hence, when $t>0$ is "small", the equations are best considered as a singular perturbation of the Darcy equations. Note that the essential boundary conditions disappear from the energy space in the limit $t=0$.

The space for the pressure is defined through the norm

$$
\|\| q \|_{t}=\sup _{\boldsymbol{v} \in \boldsymbol{V}} \frac{\langle\boldsymbol{v}, \nabla q\rangle}{\|\boldsymbol{v}\|_{t}}
$$

where $\langle\cdot, \cdot\rangle$ denotes the duality pairing in $\boldsymbol{V} \times \boldsymbol{V}^{*}$. In other words, the distributional gradient of the pressure is required to lie in the dual $\boldsymbol{V}^{*}$. The space is denoted by $Q$ :

$$
Q=\left\{q \in L_{0}^{2}(\Omega)|\|\| q \||_{t}<\infty\right\} .
$$

Note that for $(\boldsymbol{v}, q) \in \boldsymbol{V} \times Q$ it holds

$$
\langle\boldsymbol{v}, \nabla q\rangle=\left\{\begin{array}{lc}
-(\operatorname{div} \boldsymbol{v}, q) & \text { for } t>0 \\
(\boldsymbol{v}, \nabla q) & \text { for } t=0
\end{array}\right.
$$

where $(\cdot, \cdot)$ denotes the $L^{2}$-inner products. For $t>0$ the Babuška-Brezzi condition []

$$
\sup _{\boldsymbol{v} \in \boldsymbol{V}} \frac{(\operatorname{div} \boldsymbol{v}, q)}{\|\boldsymbol{v}\|_{1}} \geq C\|q\|_{0} \quad \forall q \in L_{0}^{2}(\Omega)
$$

implies that $Q=L_{0}^{2}(\Omega)$, but again the equivalence is not uniformly valid. For $0<t<C$ we have

$$
C_{1}\|q\|_{0} \leq\|\| q\|\|_{t} \leq C_{2} t^{-1}\|q\|_{0}
$$

For $t=0$ we have

$$
\|q\|_{t} \equiv\|\nabla q\|_{0}
$$

and $Q=H^{1}(\Omega) \cap L_{0}^{2}(\Omega)$.

Define the bilinear forms

$$
\begin{aligned}
a(\boldsymbol{u}, \boldsymbol{v}) & =t^{2}(\varepsilon(\boldsymbol{u}), \varepsilon(\boldsymbol{v}))+(\boldsymbol{u}, \boldsymbol{v}) \\
b(\boldsymbol{v}, p) & =\langle\boldsymbol{v}, \nabla p\rangle
\end{aligned}
$$


and

$$
\mathcal{B}(\boldsymbol{u}, p ; \boldsymbol{v}, q)=a(\boldsymbol{u}, \boldsymbol{v})+b(\boldsymbol{v}, p)+b(\boldsymbol{u}, q) .
$$

The weak formulation of the problem is then: Find $(\boldsymbol{u}, p) \in \boldsymbol{V} \times Q$ such that

$$
\mathcal{B}(\boldsymbol{u}, p ; \boldsymbol{v}, q)=\mathcal{L}(\boldsymbol{v}, q) \quad \forall(\boldsymbol{v}, q) \in \boldsymbol{V} \times Q
$$

where

$$
\mathcal{L}(\boldsymbol{v}, q)=(\boldsymbol{f}, \boldsymbol{v})-(g, q)
$$

By definition of the norms and Korn's inequality, Brezzi's conditions for a saddle point problem are satisfied, namely

$$
a(\boldsymbol{v}, \boldsymbol{v}) \geq C\|\boldsymbol{v}\|_{t}^{2} \quad \forall \boldsymbol{v} \in \boldsymbol{V} \quad \text { and } \quad \sup _{\boldsymbol{v} \in \boldsymbol{V}} \frac{b(\boldsymbol{v}, q)}{\|\boldsymbol{v}\|_{t}} \geq\|\| q \|_{t} \quad \forall q \in Q .
$$

These two imply the stability condition

$$
\sup _{(\boldsymbol{v}, q) \in \boldsymbol{V} \times Q} \frac{\mathcal{B}(\boldsymbol{w}, r ; \boldsymbol{v}, q)}{\|\boldsymbol{v}\|_{t}+\mid\|q\|_{t}} \geq C\left(\|\boldsymbol{w}\|_{t}+\mid\|r\| \|_{t}\right) \quad \forall(\boldsymbol{w}, r) \in \boldsymbol{V} \times Q
$$

by which the solution is unique.

\section{Mixed finite element methods}

We assume a partitioning $\mathcal{C}_{h}$ of the domain $\Omega$ into simplices. With $K \in \mathcal{C}_{h}$ we denote an element of the partitioning, and the maximum size of $K \in \mathcal{C}_{h}$ is denoted by $h$. With $\Gamma_{h}$ we denote the internal edges/faces of the partitioning.

The finite element spaces are a generalization of the classical MINI element [4] and they are defined as

$$
\begin{aligned}
\boldsymbol{V}_{h} & =\left\{\boldsymbol{v} \in \boldsymbol{V} \cap[C(\Omega)]^{N}|\boldsymbol{v}|_{K} \in\left[P_{k}(K) \cup B_{k+N}(K)\right]^{N} \forall K \in \mathcal{C}_{h}\right\} \\
Q_{h} & =\left\{q \in L_{0}^{2}(\Omega) \cap C(\Omega)|q|_{K} \in P_{k}(K) \forall K \in \mathcal{C}_{h}\right\}
\end{aligned}
$$

where $P_{k}(K)$ denotes the polynomials of degree $k$ and

$$
B_{k+N}(K)=P_{k+N}(K) \cap H_{0}^{1}(K)
$$

are the bubbles of degree $k+N$. In the analysis will also use the subspace $\overline{\boldsymbol{V}}_{h} \subset \boldsymbol{V}_{h}$ where the "bubbles" are left out:

$$
\overline{\boldsymbol{V}}_{h}=\left\{\boldsymbol{v} \in \boldsymbol{V} \cap[C(\Omega)]^{N}|\boldsymbol{v}|_{K} \in\left[P_{k}(K)\right]^{N} \forall K \in \mathcal{C}_{h}\right\}
$$

The finite element formulations is: find $\left(\boldsymbol{u}_{h}, p_{h}\right) \in \boldsymbol{V}_{h} \times Q_{h}$ such that

$$
\mathcal{B}\left(\boldsymbol{u}_{h}, p_{h} ; \boldsymbol{v}, q\right)=\mathcal{L}(\boldsymbol{v}, q) \quad \forall(\boldsymbol{v}, q) \in \boldsymbol{V}_{h} \times Q_{h}
$$




\subsection{Stability}

To prove the stability of our formulation we have to verify the two conditions, the ellipticity and the inf-sup condition. For this we will utilize the following discrete counterpart of the norm (9)

$$
\|\| q\left\|_{t, h}^{2}=\sum_{K \in \mathcal{C}_{h}} \frac{h_{K}^{2}}{t^{2}+h_{K}^{2}}\right\| \nabla q \|_{0, K}^{2}
$$

This norm is also important in practice, since it can be readily computed.

First, we prove the inf-sup condition with this norm.

Lemma 1. There is a constant $C>0$ such that

$$
\sup _{v \in \boldsymbol{V}_{h}} \frac{b(\boldsymbol{v}, q)}{\|\boldsymbol{v}\|_{t}} \geq C \mid\|q\|_{t, h} \quad \forall q \in Q_{h} .
$$

Proof. For $q \in Q_{h}$ given, it holds $\left.\nabla q\right|_{K} \in\left[P_{k-1}(K)\right]^{N}$, and we can define $\boldsymbol{v} \in \boldsymbol{V}_{h}$ through

$$
\left.\boldsymbol{v}\right|_{K}=\left.\left(\frac{h_{K}^{2}}{t^{2}+h_{K}^{2}}\right) b_{K} \nabla q\right|_{K}
$$

where $b_{K}$ is the cubic/quartic bubble on $K$. For $\boldsymbol{v}$ it holds

$$
b(\boldsymbol{v}, q)=(\boldsymbol{v}, \nabla q) \geq C \sum_{K \in \mathcal{C}_{h}} \frac{h_{K}^{2}}{t^{2}+h_{K}^{2}}\|\nabla q\|_{0, K}^{2}=C \mid\|q\| \|_{t, h}^{2}
$$

and

$$
\begin{aligned}
\|\boldsymbol{v}\|_{t}^{2} & =t^{2}\|\nabla \boldsymbol{v}\|_{0}^{2}+\|\boldsymbol{v}\|_{0}^{2} \leq C \sum_{K \in \mathcal{C}_{h}}\left(t^{2} h_{K}^{-2}+1\right)\|\boldsymbol{v}\|_{0, K}^{2} \\
& \leq C \sum_{K \in \mathcal{C}_{h}}\left(t^{2} h_{K}^{-2}+1\right)\left(\frac{h_{K}^{2}}{t^{2}+h_{K}^{2}}\right)^{2}\|\nabla q\|_{0, K}^{2}=C \mid\|q\| \|_{t, h}^{2} .
\end{aligned}
$$

Combining equations (29) and (30) completes the proof.

Next, we use the 'Pitkäranta-Verfürth'-trick (see $[14,17])$ to prove the stability in the continuous norm.

Lemma 2. There is a constant $C>0$ such that

$$
\sup _{\boldsymbol{v} \in \boldsymbol{V}_{h}} \frac{b(\boldsymbol{v}, q)}{\|\boldsymbol{v}\|_{t}} \geq C \mid\|q\|_{t} \quad \forall q \in Q_{h}
$$

Proof. Due to the continuous inf-sup condition (20), there exist $\boldsymbol{w} \in \boldsymbol{V}$ such that

$$
b(\boldsymbol{w}, q) \geq \mid\|q\|_{t}^{2} \quad \text { and } \quad\|\boldsymbol{w}\|_{t} \leq\|\| q\|\|_{t} \quad \forall q \in Q_{h} .
$$


With $\tilde{\boldsymbol{w}} \in \overline{\boldsymbol{V}}_{h}$ we denote the Clément-Scott-Zhang interpolant $[6,5]$ of $\boldsymbol{w}$. For this it holds

$$
\begin{aligned}
& \sum_{K \in K_{h}} h_{K}^{-2}\|\boldsymbol{w}-\tilde{\boldsymbol{w}}\|_{0, K}^{2} \leq C\|\nabla \boldsymbol{w}\|_{0}^{2}, \\
& \|\tilde{\boldsymbol{w}}\|_{0} \leq C\|\boldsymbol{w}\|_{0} \quad \text { and } \quad\|\nabla \tilde{\boldsymbol{w}}\|_{0} \leq C\|\nabla \boldsymbol{w}\|_{0} .
\end{aligned}
$$

This gives

$$
\begin{aligned}
\sum_{K \in \mathcal{C}_{h}}\left(\frac{t+h_{K}}{h_{K}}\right)^{2}\|\tilde{\boldsymbol{w}}-\boldsymbol{w}\|_{0, K}^{2} & \leq 2 \sum_{K \in \mathcal{C}_{h}}\left(\left(\frac{t}{h_{K}}\right)^{2}+1\right)\|\tilde{\boldsymbol{w}}-\boldsymbol{w}\|_{0, K}^{2} \\
& \leq C\|\boldsymbol{w}\|_{t}^{2} \leq C\|\tilde{\boldsymbol{w}}\|_{t}^{2} .
\end{aligned}
$$

Using the estimates above, we obtain

$$
\begin{aligned}
b(\tilde{\boldsymbol{w}}, q) & =(\tilde{\boldsymbol{w}}, \nabla q) \\
& =(\boldsymbol{w}, \nabla q)+(\tilde{\boldsymbol{w}}-\boldsymbol{w}, \nabla q) \\
& \geq|\|q\||_{t}^{2}-\sum_{K \in \mathcal{C}_{h}} \frac{h_{K}}{t+h_{K}}\|\nabla q\|_{0, K} \frac{t+h_{K}}{h_{K}}\|\tilde{\boldsymbol{w}}-\boldsymbol{w}\|_{0, K} \\
& \geq|\|q\||_{t}^{2}-|\|q\||_{t, h}\left(\sum_{K \in \mathcal{C}_{h}}\left(\frac{t+h_{K}}{h_{K}}\right)^{2}\|\tilde{\boldsymbol{w}}-\boldsymbol{w}\|_{0, K}^{2}\right)^{1 / 2} \\
& \geq|\|q\||_{t}^{2}-C|\|q\||_{t, h}\|\boldsymbol{w}\|_{t} \\
& \geq\left(|\|q\||_{t}-C|\|q\||_{t, h}\right)\|\boldsymbol{w}\|_{t} \\
& \geq\left(C_{1}\left|\|q\|_{t}-C_{2}\right|\|q\| \|_{t, h}\right)\|\tilde{\boldsymbol{w}}\|_{t} .
\end{aligned}
$$

Thus, we have

$$
\sup _{\boldsymbol{v} \in \boldsymbol{V}_{h}} \frac{b(\boldsymbol{v}, q)}{\|\boldsymbol{v}\|_{t}} \geq C_{1}|\|q\||_{t}-C_{2} \mid\|q\|_{t, h}
$$

Combining this estimate and Lemma 1 , with $0<\alpha<1$, we get

$$
\begin{aligned}
\sup _{\boldsymbol{v} \in \boldsymbol{V}_{h}} \frac{b(\boldsymbol{v}, q)}{\|\boldsymbol{v}\|_{t}} & =\alpha \sup _{\boldsymbol{v} \in \boldsymbol{V}_{h}} \frac{b(\boldsymbol{v}, q)}{\|\boldsymbol{v}\|_{t}}+(1-\alpha) \sup _{\boldsymbol{v} \in \boldsymbol{V}_{h}} \frac{b(\boldsymbol{v}, q)}{\|\boldsymbol{v}\|_{t}} \\
& \geq \alpha C_{1}|\|q\||_{t}-\alpha C_{2}|\|q\||_{t, h}+(1-\alpha) C|\|q\||_{t, h} \\
& =\alpha C_{1}|\|q\||_{t}+\left(C-\alpha\left(C+C_{2}\right)\right)\|\| q \|\left.\right|_{t, h} .
\end{aligned}
$$

Choosing $\alpha$ such that $0<\alpha<C /\left(C+C_{2}\right)$ proves the assertion.

Lemmas 1 and 2 give the two stability results.

Theorem 3. There is a constant $C>0$ such that

$$
\sup _{(\boldsymbol{v}, q) \in \boldsymbol{V}_{h} \times Q_{h}} \frac{\mathcal{B}(\boldsymbol{w}, r ; \boldsymbol{v}, q)}{\|\boldsymbol{v}\|_{t}+\mid\|q\|_{t}} \geq C\left(\|\boldsymbol{w}\|_{t}+\mid\|r\|_{t}\right) \quad \forall(\boldsymbol{w}, r) \in \boldsymbol{V}_{h} \times Q_{h} .
$$

Theorem 4. There is a constant $C>0$ such that

$$
\sup _{(\boldsymbol{v}, q) \in \boldsymbol{V}_{h} \times Q_{h}} \frac{\mathcal{B}(\boldsymbol{w}, r ; \boldsymbol{v}, q)}{\|\boldsymbol{v}\|_{t}+\mid\|q\|_{t, h}} \geq C\left(\|\boldsymbol{w}\|_{t}+\mid\|r\|_{t, h}\right) \quad \forall(\boldsymbol{w}, r) \in \boldsymbol{V}_{h} \times Q_{h} .
$$




\section{$3.2 \quad$ A priori estimate}

The stability estimate of Theorem 3 and the consistency gives the following quasioptimality result.

Theorem 5. There exists a constant $C>0$ such that

$$
\left\|\boldsymbol{u}-\boldsymbol{u}_{h}\right\|_{t}+\left|\left\|p-p_{h}\right\|\right|_{t} \leq C\left\{\inf _{\boldsymbol{v} \in \boldsymbol{V}_{h}}\|\boldsymbol{u}-\boldsymbol{v}\|_{t}+\inf _{q \in Q_{h}}|\|p-q\||_{t}\right\} .
$$

Standard interpolation estimates then give.

Theorem 6. Assume that the problem has a smooth solution. Then it holds

$$
\left\|\boldsymbol{u}-\boldsymbol{u}_{h}\right\|_{t}+\left|\left\|p-p_{h}\right\|\right|_{t, h}=\mathcal{O}\left(h^{k}\right) .
$$

When measuring the error in the computable mesh dependent norm for the pressure we get the following theorem.

Theorem 7. There exists $C>0$ such that

$$
\begin{aligned}
\left\|\boldsymbol{u}-\boldsymbol{u}_{h}\right\|_{t}+\left|\left\|p-p_{h}\right\|\right|_{t, h} \leq C\left(\inf _{\boldsymbol{v} \in \boldsymbol{V}_{h}}\left\{\|\boldsymbol{u}-\boldsymbol{v}\|_{t}+t\left(\sum_{K \in \mathcal{C}_{h}} h_{K}^{-2}\|\boldsymbol{u}-\boldsymbol{v}\|_{0, K}^{2}\right)^{1 / 2}\right\}\right. \\
\left.+\inf _{q \in Q_{h}}\left\{\left.\|p-q\|\right|_{t, h}+\mid\|p-q\| \|_{t}\right\}\right) .
\end{aligned}
$$

Proof. By the triangle inequality

$$
\left\|\boldsymbol{u}-\boldsymbol{u}_{h}\right\|_{t}+\left|\left\|p-p_{h}\right\|\right|_{t, h} \leq\|\boldsymbol{u}-\boldsymbol{v}\|_{t}+|\|p-q\||_{t, h}+\left\|\boldsymbol{u}_{h}-\boldsymbol{v}\right\|_{t}+\left|\left\|p_{h}-q\right\|\right|_{t, h} .
$$

Hence, we have to bound

$$
\left\|\boldsymbol{u}_{h}-\boldsymbol{v}\right\|_{t}+\left|\left\|p_{h}-q\right\|\right|_{t, h}
$$

Using the stability estimate of Theorem 4 we know there exists $(\boldsymbol{w}, r) \in$ $\boldsymbol{V}_{h} \times Q_{h}$, with

$$
\|\boldsymbol{w}\|_{t}+\mid\|r\|_{t, h} \leq C
$$

such that

$$
\left\|\boldsymbol{u}_{h}-\boldsymbol{v}\right\|_{t}+\left|\left\|p_{h}-q\right\|\right|_{t, h} \leq \mathcal{B}\left(\boldsymbol{u}_{h}-\boldsymbol{v}, p_{h}-q ; \boldsymbol{w}, r\right) .
$$

By the consistency we have

$$
\mathcal{B}\left(\boldsymbol{u}_{h}-\boldsymbol{v}, p_{h}-q ; \boldsymbol{w}, r\right)=\mathcal{B}(\boldsymbol{u}-\boldsymbol{v}, p-q ; \boldsymbol{w}, r),
$$

Using Schwartz inequality we then get

$$
\begin{aligned}
& \mathcal{B}(\boldsymbol{u}-\boldsymbol{v}, p-q ; \boldsymbol{w}, r) \\
& =t^{2}(\nabla(\boldsymbol{u}-\boldsymbol{v}), \nabla \boldsymbol{w})+(\boldsymbol{u}-\boldsymbol{v}, \boldsymbol{w})+\langle\boldsymbol{w}, p-q\rangle+(\boldsymbol{u}-\boldsymbol{v}, \nabla r) \\
& \leq t\|\nabla(\boldsymbol{u}-\boldsymbol{v})\|_{0} t\|\nabla \boldsymbol{w}\|_{0}+\|\boldsymbol{u}-\boldsymbol{v}\|_{0}\|\boldsymbol{w}\|_{0}+\|\boldsymbol{w}\|_{t}\|\|_{p}-q \|_{t} \\
& \quad+\left(\sum_{K \in \mathcal{C}_{h}}\left(\frac{t+h_{K}}{h_{K}}\right)^{2}\|\boldsymbol{u}-\boldsymbol{v}\|_{0, K}^{2}\right)^{1 / 2}\left(\sum_{K \in \mathcal{C}_{h}}\left(\frac{h_{K}}{t+h_{K}}\right)\|\nabla r\|_{0, K}^{2}\right)^{1 / 2} \\
& \leq C\left(\|\boldsymbol{u}-\boldsymbol{v}\|_{t}+\|\| p-q \|_{t}+t\left(\sum_{K \in \mathcal{C}_{h}} h_{K}^{-2}\|\boldsymbol{u}-\boldsymbol{v}\|_{0, K}^{2}\right)^{1 / 2}\right) .
\end{aligned}
$$

Combining equations (44) - (48) proves equation (43). 


\subsection{A posteriori estimate}

In this section we will introduce and analyze a residual based a posteriori estimator. In an earlier paper we have done this for the related scalar reaction-diffusion [10]. The element wise estimator is defined by

$$
\begin{aligned}
E_{K}\left(\boldsymbol{u}_{h}, p_{h}\right)^{2} & =\frac{h_{K}^{2}}{t^{2}+h_{K}^{2}}\left\|t^{2} \boldsymbol{A} \boldsymbol{u}_{h}-\boldsymbol{u}_{h}-\nabla p_{h}+\boldsymbol{f}\right\|_{0, K}^{2}+\left(t^{2}+h_{K}^{2}\right)\left\|\operatorname{div} \boldsymbol{u}_{h}-g\right\|_{0, K}^{2} \\
& +\frac{h_{K}}{t^{2}+h_{K}^{2}}\left\|\llbracket t^{2} \boldsymbol{\varepsilon}_{n}\left(\boldsymbol{u}_{h}\right) \rrbracket\right\|_{0, \partial K \backslash \partial \Omega}^{2}+\frac{t^{2}+h_{K}^{2}}{h_{K}}\left\|\boldsymbol{u}_{h} \cdot \boldsymbol{n}\right\|_{0, \partial K \cap \partial \Omega}^{2}
\end{aligned}
$$

and the global estimator is

$$
\eta=\left(\sum_{K \in \mathcal{C}_{h}} E_{K}\left(\boldsymbol{u}_{h}, p_{h}\right)^{2}\right)^{1 / 2} .
$$

Here $\varepsilon_{n}(\cdot)$ denotes the normal derivative and $\llbracket \cdot \rrbracket$ is the jump. Note, that the last term in (49) vanishes when $t>0$.

In the limit $t=0$ (or as $t<h$ ) the a posteriori estimator becomes

$E_{K}\left(\boldsymbol{u}_{h}, p_{h}\right)^{2} \approx\left\|\boldsymbol{u}_{h}+\nabla p_{h}-\boldsymbol{f}\right\|_{0, K}^{2}+h_{K}^{2}\left\|\operatorname{div} \boldsymbol{u}_{h}-g\right\|_{0, K}^{2}+h_{E}\left\|\boldsymbol{u}_{h} \cdot \boldsymbol{n}\right\|_{0, \partial K \cap \partial \Omega}^{2}$

which is the estimator for the Darcy problem. On the other hand, if $t \geq C>$ 0 , the estimator can be expressed as (since $\left.\boldsymbol{u}_{h}\right|_{\partial \Omega}=\mathbf{0}$ )

$$
\begin{aligned}
E_{K}\left(\boldsymbol{u}_{h}, p_{h}\right)^{2} \approx & h_{K}^{2}\left\|t^{2} \boldsymbol{A} \boldsymbol{u}_{h}-\boldsymbol{u}_{h}-\nabla p_{h}+\boldsymbol{f}\right\|_{0, K}^{2}+\left\|\operatorname{div} \boldsymbol{u}_{h}-g\right\|_{0, K}^{2} \\
& +h_{E}\left\|\llbracket \boldsymbol{\varepsilon}_{n}\left(\boldsymbol{u}_{h}\right) \rrbracket\right\|_{0, \partial K \backslash \partial \Omega}^{2},
\end{aligned}
$$

which is the standard Stokes estimator.

For our analysis we will need a saturation assumption. The partitioning $\mathcal{C}_{h}$ is refined into $\mathcal{C}_{h / 2}$ by dividing each triangle/tetrahedron $K$ into four/eight elements with mesh size less or equal to $h_{K} / 2$. By $\left(\boldsymbol{u}_{h / 2}, p_{h / 2}\right) \in \boldsymbol{V}_{h / 2} \times Q_{h / 2}$ we denote the finite element solution on the refined mesh.

Assumption 8. There exists a positive constant $\beta<1$ such that

$$
\left\|\boldsymbol{u}-\boldsymbol{u}_{h / 2}\right\|_{t}+\left.\left|\| p-p_{h / 2}\right|\right|_{t, h} \leq \beta\left(\left\|\boldsymbol{u}-\boldsymbol{u}_{h}\right\|_{t}+\left|\left\|p-p_{h}\right\|\right|_{t, h}\right) .
$$

The main result is the following theorem.

Theorem 9. Let Assumption 8 hold. Then there exists $C>0$ such that

$$
\left\|\boldsymbol{u}-\boldsymbol{u}_{h}\right\|_{t}+\mid\left\|p-p_{h}\right\|_{t, h} \leq C \eta
$$

Proof. By the triangle inequality the saturation assumption gives

$$
\left\|\boldsymbol{u}-\boldsymbol{u}_{h}\right\|_{t}+\left|\left\|p-p_{h}\right\|\right|_{t, h} \leq \frac{1}{1-\beta}\left(\left\|\boldsymbol{u}_{h / 2}-\boldsymbol{u}_{h}\right\|_{t}+\left|\left\|p_{h / 2}-p_{h}\right\|\right|_{t, h}\right) .
$$


From the stability, Theorem 4 , there exists $(\boldsymbol{v}, q) \in \boldsymbol{V}_{h / 2} \times Q_{h / 2}$, with

$$
\|\boldsymbol{v}\|_{t}+|\|q\||_{t, h} \leq C
$$

such that

$$
\left\|\boldsymbol{u}_{h / 2}-\boldsymbol{u}_{h}\right\|_{t}+\left|\left\|p_{h / 2}-p_{h}\right\|\right|_{t, h} \leq \mathcal{B}\left(\boldsymbol{u}_{h / 2}-\boldsymbol{u}_{h}, p_{h / 2}-p_{h} ; \boldsymbol{v}, q\right) .
$$

Let now $(\tilde{\boldsymbol{v}}, \tilde{q}) \in \overline{\boldsymbol{V}}_{h} \times Q_{h}$ be the normal Lagrange interpolants to $(\boldsymbol{v}, q)$. Since $\overline{\boldsymbol{V}}_{h} \subset \boldsymbol{V}_{h}$ and $\overline{\boldsymbol{V}}_{h} \subset \boldsymbol{V}_{h / 2}$ (and $Q_{h / 2} \subset Q_{h}$ ) it holds

$$
\mathcal{B}\left(\boldsymbol{u}_{h / 2}-\boldsymbol{u}_{h}, p_{h / 2}-p_{h} ; \tilde{\boldsymbol{v}}, \tilde{q}\right)=0 .
$$

Hence we have

$$
\mathcal{B}\left(\boldsymbol{u}_{h / 2}-\boldsymbol{u}_{h}, p_{h / 2}-p_{h} ; \boldsymbol{v}, q\right)=\mathcal{B}\left(\boldsymbol{u}_{h / 2}-\boldsymbol{u}_{h}, p_{h / 2}-p_{h} ; \boldsymbol{v}-\tilde{\boldsymbol{v}}, q-\tilde{q}\right) .
$$

Writing out the right hand side, using the fact that $\left(\boldsymbol{u}_{h / 2}, p_{h / 2}\right)$ satisfies

$$
\mathcal{B}\left(\boldsymbol{u}_{h / 2}, p_{h / 2} ; \boldsymbol{v}-\tilde{\boldsymbol{v}}, q-\tilde{q}\right)=(\boldsymbol{f}, \boldsymbol{v}-\tilde{\boldsymbol{v}})-(g, q-\tilde{q})
$$

and integrating by parts, we have

$$
\begin{aligned}
\mathcal{B} & \left(\boldsymbol{u}_{h / 2}-\boldsymbol{u}_{h}, p_{h / 2}-p_{h} ; \boldsymbol{v}-\tilde{\boldsymbol{v}}, q-\tilde{q}\right) \\
= & (\boldsymbol{f}, \boldsymbol{v}-\tilde{\boldsymbol{v}})-(g, q-\tilde{q})-t^{2}\left(\boldsymbol{\varepsilon}\left(\boldsymbol{u}_{h}\right), \boldsymbol{\varepsilon}(\boldsymbol{v}-\tilde{\boldsymbol{v}})\right)-\left(\boldsymbol{u}_{h}, \boldsymbol{v}-\tilde{\boldsymbol{v}}\right) \\
& -\left(\boldsymbol{v}-\tilde{\boldsymbol{v}}, \nabla p_{h}\right)-\left(\boldsymbol{u}_{h}, \nabla(q-\tilde{q})\right) \\
= & \sum_{K \in \mathcal{C}_{h}}\left\{\left(t^{2} \boldsymbol{A} \boldsymbol{u}_{h}-\boldsymbol{u}_{h}-\nabla p_{h}+\boldsymbol{f}, \boldsymbol{v}-\tilde{\boldsymbol{v}}\right)_{K}+t^{2}\left\langle\boldsymbol{\varepsilon}_{h}\left(\boldsymbol{u}_{h}\right), \boldsymbol{v}-\tilde{\boldsymbol{v}}\right\rangle_{\partial K}\right. \\
& \left.+\left(\operatorname{div} \boldsymbol{u}_{h}-g, q-\tilde{q}\right)_{K}-\left\langle\boldsymbol{u}_{h} \cdot \boldsymbol{n}, q-\tilde{q}\right\rangle_{\partial K \cap \partial \Omega}\right\} .
\end{aligned}
$$

Since, $\boldsymbol{v}, \tilde{\boldsymbol{v}}, q$ and $\tilde{q}$, all are in finite element subspaces, scaling arguments give

$$
\begin{aligned}
\left(\sum_{K \in \mathcal{C}_{h}}\left(\frac{t+h_{K}}{h_{K}}\right)^{2}\|\boldsymbol{v}-\tilde{\boldsymbol{v}}\|_{0, K}^{2}\right)^{1 / 2} & \leq C\left(\sum_{K \in \mathcal{C}_{h}}\left(t^{2}\|\nabla \boldsymbol{v}\|_{0, K}^{2}+\|\boldsymbol{v}\|_{0, K}^{2}\right)\right)^{1 / 2} \\
& \leq C\|\boldsymbol{v}\|_{t} \leq C \\
\left(\sum_{K \in \mathcal{C}_{h}} \frac{t^{2}+h_{K}^{2}}{h_{K}}\|\boldsymbol{v}-\tilde{\boldsymbol{v}}\|_{0, \partial K}^{2}\right)^{1 / 2} & \leq C\left(\sum_{K \in \mathcal{C}_{h}} \frac{t^{2}+h_{K}^{2}}{h_{K}} h_{K}^{-1}\|\boldsymbol{v}-\tilde{\boldsymbol{v}}\|_{0, K}^{2}\right)^{1 / 2} \\
& =C\left(\sum_{K \in \mathcal{C}_{h}}\left(\frac{t^{2}}{h_{K}^{2}}+1\right)\|\boldsymbol{v}-\tilde{\boldsymbol{v}}\|_{0, K}^{2}\right)^{1 / 2} \\
& \leq C\left(\sum_{K \in \mathcal{C}_{h}}\left(t^{2}\|\nabla \boldsymbol{v}\|_{0, K}^{2}+\|\boldsymbol{v}\|_{0, K}^{2}\right)\right) \\
& =C\|\boldsymbol{v}\|_{t} \leq C
\end{aligned}
$$


and

$$
\begin{aligned}
& \left(\sum_{K \in \mathcal{C}_{h}}\left(\frac{h_{K}}{\left(t+h_{K}\right)^{2}}\|q-\tilde{q}\|_{0, \partial K}^{2}+\left(t+h_{K}\right)^{-2}\|q-\tilde{q}\|_{0, K}^{2}\right)\right)^{1 / 2} \\
& \leq C\left(\sum_{K \in \mathcal{C}_{h}}\left(\frac{h_{K}}{t+h_{K}}\right)^{2}\|\nabla q\|_{0, K}^{2}\right)^{1 / 2} \leq C \mid\|q\| \|_{t, h} \leq C
\end{aligned}
$$

Using the Schwartz inequality and the properties above in equation (58) completes the proof.

We show that the a posteriori estimator also gives a lower bound to the error. In this sense the estimator is sharp.

\subsection{Efficiency of the a posteriori estimate}

We show that the a posteriori upper bound is also a lower bound to the error. In this sense the estimator is sharp.

Theorem 10. There exist $C>0$ such that

$$
\begin{aligned}
C \eta^{2} \leq & \left\|\boldsymbol{u}-\boldsymbol{u}_{h}\right\|_{t}^{2}+\mid\left\|p-p_{h}\right\|_{t, h}^{2} \\
& +\sum_{K \in \mathcal{C}_{h}}\left(\frac{h_{K}^{2}}{t^{2}+h_{K}^{2}}\left\|\boldsymbol{f}-\boldsymbol{f}_{h}\right\|_{0, K}^{2}+\left(t^{2}+h_{K}^{2}\right)\left\|g-g_{h}\right\|_{0, K}^{2}\right)
\end{aligned}
$$

where the projections $\boldsymbol{f}_{h} \in \boldsymbol{V}_{h}$ and $g_{h} \in Q_{h}$.

We use suitable cut-off functions to prove the above theorem, we refer to [18] for more details. The first cut-off function is $\Psi_{K}$; the support of $\Psi_{K}$ is element $K$ and $0 \leq \Psi_{K} \leq 1$. The second cut-off function is $\Psi_{E}$; the support of $\Psi_{E}$ is $\omega_{E}$ and $0 \leq \Psi_{E} \leq 1$. The domain $\omega_{E}$ is the elements sharing edge (in $3 \mathrm{D}$ face) $E$. For the edge (or face) $E$ we also need an extension mapping $\chi: L^{2}(E) \rightarrow L^{2}\left(\omega_{E}\right)$ such that in $E \chi$ is the identity operator. The proof of the lemma below follows with scaling arguments; note that $p$ and $\sigma$ are polynomials, cf. [18].

Lemma 11. For an arbitrary element $K$, having edge/face $E$, and for arbitrary polynomials $p$ and $\sigma$ it holds:

$$
\begin{aligned}
\left\|\Psi_{K} p\right\|_{0, K} & \leq\|p\|_{0, K} \leq C\left\|\Psi_{K}^{1 / 2} p\right\|_{0, K} \\
\left\|\nabla\left(\Psi_{K} p\right)\right\|_{0, K} & \leq C h_{K}^{-1}\left\|\Psi_{K} p\right\|_{0, K} \\
\|\sigma\|_{0, E} & \leq C\left\|\Psi_{E}^{1 / 2} \sigma\right\|_{0, E} \\
C h_{E}^{1 / 2}\|\sigma\|_{0, E} & \leq\left\|\Psi_{E} \chi \sigma\right\|_{0, E} \leq C h_{E}^{1 / 2}\|\sigma\|_{0, E} \\
\left\|\nabla\left(\Psi_{E} \chi \sigma\right)\right\|_{0, K} & \leq C h_{K}^{-1}\left\|\Psi_{E} \chi \sigma\right\|_{0, K} .
\end{aligned}
$$


Proof. We bound the terms of $E_{K}\left(\boldsymbol{u}_{h}, p_{h}\right)$ separately. We begin with the first internal residual term and introduce

$$
\begin{aligned}
R_{K}^{1} & =t^{2} \boldsymbol{A} \boldsymbol{u}_{h}-\boldsymbol{u}_{h}-\nabla p_{h}+\boldsymbol{f}, \quad R_{K, \text { red }}^{1}=t^{2} \boldsymbol{A} \boldsymbol{u}_{h}-\boldsymbol{u}_{h}-\nabla p_{h}+\boldsymbol{f}_{h}, \\
\boldsymbol{w} & =\Psi_{K} R_{K, \text { red }}^{1}
\end{aligned}
$$

We have, using Lemma 11,

$$
\begin{aligned}
& \left\|R_{K, \mathrm{red}}^{1}\right\|_{0, K}^{2} \leq C\left\|\Psi_{K}^{1 / 2} R_{K, \mathrm{red}}^{1}\right\|_{0, K}^{2}=C\left(R_{K, \mathrm{red}}^{1}, \boldsymbol{w}\right)_{K} \\
& =C\left(\left(R_{K}^{1}, \boldsymbol{w}\right)_{K}+\left(\boldsymbol{f}_{h}-\boldsymbol{f}, \boldsymbol{w}\right)_{K}\right) \\
& =C\left(t^{2}\left(\nabla\left(\boldsymbol{u}-\boldsymbol{u}_{h}\right), \nabla \boldsymbol{w}\right)_{K}+\left(\boldsymbol{u}_{h}-\boldsymbol{u}, \boldsymbol{w}\right)_{K}\right. \\
& \left.\quad+\left(\nabla\left(p_{h}-p\right), \boldsymbol{w}\right)_{K}+\left(\boldsymbol{f}_{h}-\boldsymbol{f}, \boldsymbol{w}\right)_{K}\right) \\
& \quad C \quad\left(t^{2} h_{K}^{-1}\left\|\nabla\left(\boldsymbol{u}-\boldsymbol{u}_{h}\right)\right\|_{0, K}+\left\|\boldsymbol{u}-\boldsymbol{u}_{h}\right\|_{0, K}\right. \\
& \left.\quad+\left\|\nabla\left(p-p_{h}\right)\right\|_{0, K}+\left\|\boldsymbol{f}-\boldsymbol{f}_{h}\right\|_{0, K}\right)\left\|R_{K, \mathrm{red}}^{1}\right\|_{0, K} .
\end{aligned}
$$

Combining the above result with $\left\|R_{K}^{1}\right\|_{0, K} \leq\left\|R_{K, \text { red }}^{1}\right\|_{0, K}+\left\|\boldsymbol{f}-\boldsymbol{f}_{h}\right\|_{0, K}$ gives

$$
\begin{aligned}
& \frac{h_{K}}{t+h_{K}}\left\|t^{2} \boldsymbol{A} \boldsymbol{u}_{h}-\boldsymbol{u}_{h}-\nabla p_{h}+\boldsymbol{f}\right\|_{0, K} \\
& \leq C\left(\left\|\boldsymbol{u}-\boldsymbol{u}_{h}\right\|_{t, K}+\left|\left\|p-p_{h}\right\|\right|_{t, h, K}+\frac{h_{K}}{t+h_{K}}\left\|\boldsymbol{f}-\boldsymbol{f}_{h}\right\|_{0, K}\right) .
\end{aligned}
$$

Next bound the second internal residual term and introduce

$$
\begin{aligned}
R_{K}^{2} & =\operatorname{div} \boldsymbol{u}_{h}-g, \quad R_{K, \text { red }}^{2}=\operatorname{div} \boldsymbol{u}_{h}-g_{h}, \\
\boldsymbol{w} & =\Psi_{K} R_{K, \text { red }}^{2} .
\end{aligned}
$$

Using Lemma 11 we get

$$
\begin{aligned}
\| & R_{K, \text { red }}^{2}\left\|_{0, K}^{2} \leq C\right\| \Psi_{K}^{1 / 2} R_{K, \text { red }}^{2} \|_{0, K}^{2}=C\left(R_{K, \text { red }}^{2}, \boldsymbol{w}\right)_{K} \\
= & C\left(\left(R_{K}^{2}, \boldsymbol{w}\right)_{K}+\left(g-g_{h}, \boldsymbol{w}\right)_{K}\right) \\
= & C\left(\left(\operatorname{div}\left(\boldsymbol{u}_{h}-\boldsymbol{u}\right), \boldsymbol{w}\right)_{K}+\left(g-g_{h}, \boldsymbol{w}\right)_{K}\right) \\
= & C\left(\frac{t}{t+h_{K}}\left(\operatorname{div}\left(\boldsymbol{u}_{h}-\boldsymbol{u}\right), \boldsymbol{w}\right)_{K}\right. \\
& \left.\quad+\frac{h_{K}}{t+h_{K}}\left(\boldsymbol{u}_{h}-\boldsymbol{u}, \operatorname{div} \boldsymbol{w}\right)_{K}+\left(g-g_{h}, \boldsymbol{w}\right)_{K}\right) \\
\leq & C\left(\left(t+h_{K}\right)^{-1}\left\|\boldsymbol{u}-\boldsymbol{u}_{h}\right\|_{t, K}+\left\|g-g_{h}\right\|_{0, K}\right)\left\|R_{K, \text { red }}^{2}\right\|_{0, K} .
\end{aligned}
$$

Combining the result with $\left\|R_{K}^{2}\right\|_{0, K} \leq\left\|R_{K, \text { red }}^{2}\right\|_{0, K}+\left\|g-g_{h}\right\|_{0, K}$ gives

$$
\left(t+h_{K}\right)\left\|\operatorname{div} \boldsymbol{u}_{h}-g\right\|_{0, K} \leq C\left(\left\|\boldsymbol{u}-\boldsymbol{u}_{h}\right\|_{t, K}+\left(t+h_{K}\right)\left\|g-g_{h}\right\|_{0, K}\right) .
$$


Next we bound the internal jumps. We introduce

$$
R_{E}^{1}=t^{2} \llbracket \varepsilon_{n}\left(\boldsymbol{u}_{h}\right) \rrbracket, \quad \boldsymbol{w}=\Psi_{E} \chi R_{E}^{1}
$$

and continue with Lemma 11

$$
\begin{aligned}
& \left\|R_{E}^{1}\right\|_{0, E}^{2} \leq C\left\|\Psi_{E}^{1 / 2} R_{E}^{1}\right\|_{0, E}^{2}=C\left(R_{E}^{1}, \boldsymbol{w}\right)_{E} \\
& =C\left(\left(R_{K}^{1}, \boldsymbol{w}\right)_{\omega_{E}}-t^{2}\left(\nabla\left(\boldsymbol{u}-\boldsymbol{u}_{h}\right), \nabla \boldsymbol{w}\right)_{\omega_{E}}\right. \\
& \left.\quad-\left(\boldsymbol{u}-\boldsymbol{u}_{h}, \boldsymbol{w}\right)_{\omega_{E}}-\left(\nabla\left(p-p_{h}\right), \boldsymbol{w}\right)_{\omega_{E}}\right) \\
& \quad \leq C\left(t^{2} h_{K}^{-1 / 2}\left\|\nabla\left(\boldsymbol{u}-\boldsymbol{u}_{h}\right)\right\|_{0, \omega_{E}}+h_{K}^{1 / 2}\left\|\boldsymbol{u}-\boldsymbol{u}_{h}\right\|_{\omega_{E}}\right. \\
& \left.\quad+h_{K}^{1 / 2}\left\|\nabla\left(p-p_{h}\right)\right\|_{\omega_{E}}+h_{K}^{1 / 2}\left\|\boldsymbol{f}-\boldsymbol{f}_{h}\right\|_{\omega_{E}}\right)\left\|R_{E}^{1}\right\|_{0, E} .
\end{aligned}
$$

Thus, we have

$$
\left.\frac{h_{E}^{1 / 2}}{t+h_{E}}\left\|t^{2} \llbracket \varepsilon_{n}\left(\boldsymbol{u}_{h}\right) \rrbracket\right\|_{0, E} \leq C\left(\left\|\boldsymbol{u}-\boldsymbol{u}_{h}\right\|_{t, \omega_{E}}+\frac{h_{K}}{t+h_{K}} \| \boldsymbol{f}-\boldsymbol{f}_{h}\right) \|_{\omega_{E}}\right) .
$$

Lastly we bound the boundary residual. We define

$$
R_{E}^{2}=\left(\boldsymbol{u}-\boldsymbol{u}_{h}\right) \cdot \boldsymbol{n}, \quad \boldsymbol{w}=\Psi_{E} \chi R_{E}^{2}
$$

and continue with Lemma 11

$$
\begin{aligned}
& \left\|R_{E}^{2}\right\|_{0, E}^{2} \leq C\left\|\Psi_{E}^{1 / 2} R_{E}^{2}\right\|_{0, E}^{2}=C\left(R_{E}^{2}, \boldsymbol{w}\right)_{E} \\
& =C\left(\left(R_{K}^{2}, \boldsymbol{w}\right)_{\omega_{E}}+\left(\boldsymbol{u}_{h}-\boldsymbol{u}, \nabla \boldsymbol{w}\right)_{\omega_{E}}\right) \\
& \leq C\left(\frac{h_{K}^{1 / 2}}{t+h_{K}}\left\|\boldsymbol{u}-\boldsymbol{u}_{h}\right\|_{t, \omega_{E}}+h_{K}^{1 / 2}\left\|g-g_{h}\right\|_{\omega_{E}}+h_{K}^{-1 / 2}\left\|\boldsymbol{u}-\boldsymbol{u}_{h}\right\|_{\omega_{E}}\right)\left\|R_{E}^{2}\right\|_{0, E} .
\end{aligned}
$$

Hence we get

$$
\frac{t+h_{K}}{h_{K}^{1 / 2}}\left\|\boldsymbol{u}-\boldsymbol{u}_{h}\right\|_{0, E} \leq C\left(\left\|\boldsymbol{u}-\boldsymbol{u}_{h}\right\|_{t, \omega_{E}}+\left(t+h_{K}\right)\left\|g-g_{h}\right\|_{\omega_{E}}\right) .
$$

Now we have bounded all the terms of the a posteriori estimator and combining equations (69), (71), (73) and (75) completes the proof.

\section{Stabilized methods}

Stabilized methods enable us to use the standard finite elements without bubble degrees of freedom. Thus, the subspaces are

$$
\begin{aligned}
\boldsymbol{V}_{h} & =\left\{\boldsymbol{v} \in \boldsymbol{V} \cap[C(\Omega)]^{N}|\boldsymbol{v}|_{K} \in\left[P_{k}(K)\right]^{N} \forall K \in \mathcal{C}_{h}\right\}, \\
Q_{h} & =\left\{q \in L_{0}^{2}(\Omega) \cap C(\Omega)|q|_{K} \in P_{k}(K) \forall K \in \mathcal{C}_{h}\right\},
\end{aligned}
$$


The stabilized method is: Find $\left(\boldsymbol{u}_{h}, p_{h}\right) \in \boldsymbol{V}_{h} \times Q_{h}$ such that

$$
\mathcal{B}_{h}\left(\boldsymbol{u}_{h}, p_{h} ; \boldsymbol{v}, q\right)=\mathcal{L}_{h}(\boldsymbol{v}, q) \quad \forall(\boldsymbol{v}, q) \in \boldsymbol{V}_{h} \times Q_{h},
$$

with

$$
\begin{aligned}
\mathcal{B}_{h}\left(\boldsymbol{u}_{h}, p_{h} ; \boldsymbol{v}, q\right)= & \mathcal{B}\left(\boldsymbol{u}_{h}, p_{h} ; \boldsymbol{v}, q\right) \\
& -\alpha \sum_{K \in \mathcal{C}_{h}} \frac{h_{K}^{2}}{t^{2}+h_{K}^{2}}\left(t^{2} \boldsymbol{A} \boldsymbol{u}_{h}-\boldsymbol{u}_{h}-\nabla p_{h}, t^{2} \boldsymbol{A} \boldsymbol{v}-\boldsymbol{v}-\nabla q\right)_{K}
\end{aligned}
$$

and

$$
\mathcal{L}_{h}(\boldsymbol{v}, q)=\mathcal{L}(\boldsymbol{v}, q)-\alpha \sum_{K \in \mathcal{C}_{h}} \frac{h_{K}^{2}}{t^{2}+h_{K}^{2}}\left(\boldsymbol{f}, t^{2} \boldsymbol{A} \boldsymbol{v}-\boldsymbol{v}-\nabla q\right)_{K},
$$

with a parameter $\alpha>0$. For the method to be consistent we assume that

$$
t^{2} \boldsymbol{A} \boldsymbol{u}-\boldsymbol{u}-\nabla p=\boldsymbol{f} \in\left[L^{2}(\Omega)\right]^{N} .
$$

Then it holds

$$
\mathcal{B}_{h}\left(\boldsymbol{u}-\boldsymbol{u}_{h}, p-p_{h} ; \boldsymbol{v}, q\right)=0 \quad \forall(\boldsymbol{v}, q) \in \boldsymbol{V}_{h} \times Q_{h} .
$$

Note, that one does not have to assume that $t^{2} \boldsymbol{A} \boldsymbol{u} \in\left[L^{2}(\Omega)\right]^{2}$, and $\nabla p \in$ $L^{2}(\Omega)$ (contrary to some quite widespread belief).

\subsection{Stability}

For the analysis it is convenient to introduce the constant $C_{I}$ in the following inverse inequality

$$
h_{K}^{2}\|\boldsymbol{A} \boldsymbol{w}\|_{0, K}^{2} \leq C_{I}\|\nabla \boldsymbol{w}\|_{0, K}^{2} \quad \forall \boldsymbol{w} \in\left[P_{k}(K)\right]^{N} .
$$

The stability result is then.

Theorem 12. Assume that $0<\alpha<\min \left\{1 /\left(2 C_{I}\right), 1 / 2\right\}$. Then there exists a constant $C>0$ such that

$$
\sup _{(\boldsymbol{v}, q) \in \boldsymbol{V}_{h} \times Q_{h}} \frac{\mathcal{B}_{h}(\boldsymbol{w}, r ; \boldsymbol{v}, q)}{\|\boldsymbol{v}\|_{t}+|\|q\||_{t, h}} \geq C\left(\|\boldsymbol{w}\|_{t}+|\|r\||_{t, h}\right) \quad \forall(\boldsymbol{w}, r) \in \boldsymbol{V}_{h} \times Q_{h} .
$$

Proof. For $(\boldsymbol{w}, r) \in \boldsymbol{V}_{h} \times Q_{h}$ arbitrary we have

$$
\begin{aligned}
\mathcal{B}_{h}(\boldsymbol{w}, r ; \boldsymbol{w},-r)= & t^{2}\|\nabla \boldsymbol{w}\|_{0}^{2}+\|\boldsymbol{w}\|_{0}^{2} \\
& -\alpha \sum_{K \in \mathcal{C}_{h}} \frac{h_{K}^{2}}{t^{2}+h_{K}^{2}}\left\{\left\|t^{2} \boldsymbol{A} \boldsymbol{w}-\boldsymbol{w}\right\|_{0, K}^{2}-\|\nabla r\|_{0, K}^{2}\right\} .
\end{aligned}
$$

From this we get

$$
\begin{aligned}
\mathcal{B}_{h}(\boldsymbol{w}, r ; \boldsymbol{w},-r) \geq & t^{2}\|\nabla \boldsymbol{w}\|_{0}^{2}+\|\boldsymbol{w}\|_{0}^{2}+\alpha|\|r\||_{t, h}^{2} \\
& -2 \alpha \sum_{K \in \mathcal{C}_{h}} \frac{h_{K}^{2}}{t^{2}+h_{K}^{2}} t^{4}\|\boldsymbol{A} \boldsymbol{w}\|_{0, K}^{2}-2 \alpha \sum_{K \in \mathcal{C}_{h}} \frac{h_{K}^{2}}{t^{2}+h_{K}^{2}}\|\boldsymbol{w}\|_{0, K}^{2} .
\end{aligned}
$$


Applying the inverse inequality gives

$$
\mathcal{B}_{h}(\boldsymbol{w}, r ; \boldsymbol{w},-r) \geq\left(1-2 \alpha C_{I}\right) t^{2}\|\nabla \boldsymbol{w}\|_{0}^{2}+(1-2 \alpha)\|\boldsymbol{w}\|_{0}^{2}+\alpha|\|r\||_{t, h}^{2} .
$$

The assumption $0<\alpha<\min \left\{1 /\left(2 C_{I}\right), 1 / 2\right\}$ implies the asserted stability.

Remark 13. Using the Pitkäranta-Verfürth technique is also possible to prove the stability with the continuous norm for the pressure

$$
\sup _{(\boldsymbol{v}, q) \in \boldsymbol{V}_{h} \times Q_{h}} \frac{\mathcal{B}_{h}(\boldsymbol{w}, r ; \boldsymbol{v}, q)}{\|\boldsymbol{v}\|_{t}+\mid\|q\|_{t}} \geq C\left(\|\boldsymbol{w}\|_{t}+|\|r\||_{t}\right) \quad \forall(\boldsymbol{w}, r) \in \boldsymbol{V}_{h} \times Q_{h} .
$$

See [7] where this is done for the Stokes problem.

\subsection{A priori estimate}

In the spirit of stabilized methods and a posteriori estimates we will formulate the a priori estimate as a quasi-optimality result that contain a term measuring the residual.

Theorem 14. Assume that $0<\alpha<\min \left\{1 /\left(2 C_{I}\right), 1 / 2\right\}$. Then it holds

$$
\begin{aligned}
& \left\|\boldsymbol{u}-\boldsymbol{u}_{h}\right\|_{t}+\left|\left\|p-p_{h}\right\|\right|_{t, h} \\
& \leq C \inf _{(\boldsymbol{v}, q) \in \boldsymbol{V}_{h} \times Q_{h}}\left\{\|\boldsymbol{u}-\boldsymbol{v}\|_{t}+t\left(\sum_{K \in \mathcal{C}_{h}} h_{K}^{-2}\|\boldsymbol{u}-\boldsymbol{v}\|_{0, K}^{2}\right)^{1 / 2}\right. \\
& \quad+|\|p-q\||_{t, h}+|\|p-q\||_{t} \\
& \left.\quad+\left(\sum_{K \in \mathcal{C}_{h}} \frac{h_{K}^{2}}{t^{2}+h_{K}^{2}}\left\|t^{2} \boldsymbol{A} \boldsymbol{v}-\boldsymbol{v}-\nabla q+\boldsymbol{f}\right\|_{0, K}^{2}\right)^{1 / 2}\right\} .
\end{aligned}
$$

Proof. The proof is very similar to the proof of Theorem 7 and here we only consider the additional terms arising from the added stabilizing term.

For equation (89) to hold, all we need to bound is

$$
I=\sum_{K \in \mathcal{C}_{h}} \frac{h_{K}^{2}}{t^{2}+h_{K}^{2}}\left(t^{2} \boldsymbol{A}(\boldsymbol{u}-\boldsymbol{v})-(\boldsymbol{u}-\boldsymbol{v})-\nabla(p-q), t^{2} \boldsymbol{A} \boldsymbol{w}-\boldsymbol{w}-\nabla r\right)_{K}
$$

Assumption (81) gives

$$
\begin{aligned}
I= & \sum_{K \in \mathcal{C}_{h}} \frac{h_{K}^{2}}{t^{2}+h_{K}^{2}}\left(-t^{2} \boldsymbol{A} \boldsymbol{v}+\boldsymbol{v}+\nabla q-\boldsymbol{f}, t^{2} \boldsymbol{A} \boldsymbol{w}-\boldsymbol{w}-\nabla r\right)_{K} \\
\leq & \left(\sum_{K \in \mathcal{C}_{h}} \frac{h_{K}^{2}}{t^{2}+h_{K}^{2}}\left\|t^{2} \boldsymbol{A} \boldsymbol{v}-\boldsymbol{v}-\nabla q+\boldsymbol{f}\right\|_{0, K}^{2}\right)^{1 / 2} \\
& \times\left(\sum_{K \in \mathcal{C}_{h}} \frac{h_{K}^{2}}{t^{2}+h_{K}^{2}}\left\|t^{2} \boldsymbol{A} \boldsymbol{w}-\boldsymbol{w}-\nabla r\right\|_{0, K}^{2}\right)^{1 / 2} .
\end{aligned}
$$


Using the inverse inequality (83) we have

$$
\begin{aligned}
& \sum_{K \in \mathcal{C}_{h}} \frac{h_{K}^{2}}{t^{2}+h_{K}^{2}}\left\|t^{2} \boldsymbol{A} \boldsymbol{w}-\boldsymbol{w}-\nabla r\right\|_{0, K}^{2} \\
& \leq C \sum_{K \in \mathcal{C}_{h}}\left(\frac{t^{2}}{t^{2}+h_{K}^{2}} t^{2} h_{K}^{2}\|\boldsymbol{A} \boldsymbol{w}\|_{0, K}^{2}+\frac{h_{K}^{2}}{t^{2}+h_{K}^{2}}\|\boldsymbol{w}\|_{0, K}^{2}+\frac{h_{K}^{2}}{t^{2}+h_{K}^{2}}\|\nabla q\|_{0, K}^{2}\right) \\
& \leq C\left(\|\boldsymbol{w}\|_{t}+\|r\|_{t, h}\right) \leq C
\end{aligned}
$$

The relations $(90)-(92)$ prove (93).

Again, standard interpolation estimates give

Theorem 15. Assume that $0<\alpha<\min \left\{1 /\left(2 C_{I}\right), 1 / 2\right\}$ and that the problem has a smooth solution. Then it holds

$$
\left\|\boldsymbol{u}-\boldsymbol{u}_{h}\right\|_{t}+\left|\left\|p-p_{h}\right\|\right|_{t, h}=\mathcal{O}\left(h^{k}\right) .
$$

\subsection{A posteriori estimate}

The a posteriori estimator is defined exactly as for the mixed method, i.e. by $(50)$.

Theorem 16. Let Assumption 8 hold. Then there exist constants $C_{1}, C_{2}>0$ such that

$$
C_{1} \eta \leq\left\|\boldsymbol{u}-\boldsymbol{u}_{h}\right\|_{t}+\left|\left\|p-p_{h}\right\|\right|_{t, h} \leq C_{2} \eta
$$

Proof. In addition to the terms estimated in Theorem 9 we get the term

$$
\left|\alpha \sum_{K \in \mathcal{C}_{h}} \frac{h_{K}^{2}}{t^{2}+h_{K}^{2}}\left(-t^{2} \boldsymbol{A} \boldsymbol{u}_{h}+\boldsymbol{u}_{h}+\nabla p_{h}-\boldsymbol{f}, t^{2} \boldsymbol{A}(\boldsymbol{v}-\tilde{\boldsymbol{v}})-(\boldsymbol{v}-\tilde{\boldsymbol{v}})-\nabla(q-\tilde{q})\right)_{K}\right| .
$$

Using the Schwarz inequality this is bounded by

$$
\begin{aligned}
& \left(\sum_{K \in \mathcal{C}_{h}} \frac{h_{K}^{2}}{t^{2}+h_{K}^{2}}\left\|t^{2} \boldsymbol{A} \boldsymbol{u}_{h}-\boldsymbol{u}_{h}-\nabla p_{h}+\boldsymbol{f}\right\|_{0, K}^{2}\right)^{1 / 2} \\
& \quad \times\left(\sum_{K \in \mathcal{C}_{h}} \frac{h_{K}^{2}}{t^{2}+h_{K}^{2}}\left\|t^{2} \boldsymbol{A}(\boldsymbol{v}-\tilde{\boldsymbol{v}})-(\boldsymbol{v}-\tilde{\boldsymbol{v}})-\nabla(q-\tilde{q})\right\|_{0, K}^{2}\right)^{1 / 2} .
\end{aligned}
$$

Noticing that

$$
\begin{aligned}
& \sum_{K \in \mathcal{C}_{h}} \frac{h_{K}^{2}}{t^{2}+h_{K}^{2}}\left\|t^{2} \boldsymbol{A}(\boldsymbol{v}-\tilde{\boldsymbol{v}})-(\boldsymbol{v}-\tilde{\boldsymbol{v}})-\nabla(q-\tilde{q})\right\|_{0, K}^{2} \\
& \leq C \sum_{K \in \mathcal{C}_{h}}\left(\frac{t^{2}}{t^{2}+h_{K}^{2}} t^{2} h_{K}^{2}\|\boldsymbol{A}(\boldsymbol{v}-\tilde{\boldsymbol{v}})\|_{0, K}^{2}+\frac{h_{K}^{2}}{t^{2}+h_{K}^{2}}\|\boldsymbol{v}-\tilde{\boldsymbol{v}}\|_{0, K}^{2}\right. \\
& \left.\quad+\frac{h_{K}^{2}}{t^{2}+h_{K}^{2}}\|\nabla(q-\tilde{q})\|_{0, K}^{2}\right) \\
& \leq C\left(t^{2}\|\nabla \boldsymbol{v}\|_{0}^{2}+\|\boldsymbol{v}\|_{0}^{2}+\|q\|_{t, h}^{2}\right) \leq C
\end{aligned}
$$


completes the proof of the upper bound.

The proof of the lower bound does not use the bilinear form. Hence the proof of Theorem 10 also holds in the present case.

\section{$5 \quad$ Imposing boundary conditions using Nitsche's method}

In this section we will outline the modified finite element methods when the Dirichlet boundary conditions are imposed in a weak sense using the technique of Nitsche [13]. Using this, we obtain formulations that uses the same finite element spaces both for $t>0$ and in the limit $t=0$. The finite element space $Q_{h}$ used for the pressure is unaltered, i.e. (23) and (77). The spaces for the velocity are altered so that no boundary conditions are assumed; a spaces including "bubbles" for the mixed formulation:

$$
\boldsymbol{V}_{h}=\left\{\boldsymbol{v} \in[C(\Omega)]^{N}|\boldsymbol{v}|_{K} \in\left[P_{k}(K) \cup B_{k+N}(K)\right]^{N} \forall K \in \mathcal{C}_{h}\right\},
$$

and a clean polynomial space for the stabilized method:

$$
\boldsymbol{V}_{h}=\left\{\boldsymbol{v} \in[C(\Omega)]^{N}|\boldsymbol{v}|_{K} \in\left[P_{k}(K)\right]^{N} \forall K \in \mathcal{C}_{h}\right\} .
$$

The discrete variational formulations are modified by changing the bilinear form $a(\cdot, \cdot)$ in $(17)$ to

$$
\begin{aligned}
a_{h}(\boldsymbol{u}, \boldsymbol{v})= & t^{2}((\boldsymbol{\varepsilon}(\boldsymbol{u}), \boldsymbol{\varepsilon}(\boldsymbol{v})) \\
& \left.+\sum_{E \in \Gamma_{h}}\left(-\left\langle\varepsilon_{n}(\boldsymbol{u}), \boldsymbol{v}\right\rangle_{E}-\left\langle\varepsilon_{n}(\boldsymbol{v}), \boldsymbol{u}\right\rangle_{E}+\gamma h_{E}^{-1}\langle\boldsymbol{u}, \boldsymbol{v}\rangle_{E}\right)\right) \\
& +(\boldsymbol{u}, \boldsymbol{v}),
\end{aligned}
$$

where we denote with $\Gamma_{h}$ the edges/faces on the boundary $\partial \Omega$. The bilinear forms obtained we denote by $\mathcal{N}_{h}$. The right hand sides, given by (19) and (80), respectively, we denote by $\mathcal{F}_{h}$. The weak formulation of the problem is then: find $\left(\boldsymbol{u}_{h}, p_{h}\right) \in \boldsymbol{V}_{h} \times Q_{h}$ such that

$$
\mathcal{N}_{h}\left(\boldsymbol{u}_{h}, p_{h} ; \boldsymbol{v}, q\right)=\mathcal{F}_{h}(\boldsymbol{v}, q) \quad \forall(\boldsymbol{v}, q) \in \boldsymbol{V}_{h} \times Q_{h} .
$$

This formulation is clearly consistent. For the analysis one uses the following norms for the velocity

$$
\begin{aligned}
& \|\boldsymbol{v}\|_{t, h}^{2}=t^{2}\left(\|\nabla \boldsymbol{v}\|_{0}^{2}+\sum_{E \in \Gamma_{h}} h_{E}^{-1}\|\boldsymbol{v}\|_{0, E}^{2}\right)+\|\boldsymbol{v}\|_{0}^{2}, \\
& \square \boldsymbol{v} \square_{t, h}^{2}=\|\boldsymbol{v}\|_{t, h}^{2}+t^{2} \sum_{E \in \Gamma_{h}} h_{E}\left\|\varepsilon_{n}(\boldsymbol{v})\right\|_{0, E}^{2} .
\end{aligned}
$$

By the discrete trace inequality (when $E \subset \partial K$ we have $h_{E} \approx h_{K}$ )

$$
h_{K}\left\|\varepsilon_{n}(\boldsymbol{v})\right\|_{0, \partial K}^{2} \leq\left. C_{I}^{\prime}\|\nabla \boldsymbol{v}\|_{0, K}^{2} \quad \forall \boldsymbol{v} \in \boldsymbol{V}_{h}\right|_{K}
$$

the two norms are equivalent in $\boldsymbol{V}_{h}$. From which the coercivity of $a_{h}$ easily follows using Schwartz and Young's inequalities [13, 16]. 
Lemma 17. For $\gamma>C_{I}^{\prime}$ it holds

$$
a_{h}(\boldsymbol{v}, \boldsymbol{v}) \geq C\|\boldsymbol{v}\|_{t, h}^{2} \quad \forall \boldsymbol{v} \in \boldsymbol{V}_{h} .
$$

The proofs of the stability of the original methods carry over the the present modifications with the norm $\|\cdot\|_{t}$ changed to $\|\cdot\|_{t, h}$.

Theorem 18. Assume that the stability parameters satisfy $\gamma>C_{I}^{\prime}$ and $0<$ $\alpha<\min \left\{1 /\left(2 C_{I}\right), 1 / 2\right\}$. Then there exists a constant $C>0$ such that

$$
\sup _{(\boldsymbol{v}, q) \in \boldsymbol{V}_{h} \times Q_{h}} \frac{\mathcal{B}_{h}(\boldsymbol{w}, r ; \boldsymbol{v}, q)}{\|\boldsymbol{v}\|_{t, h}+|\|q\||_{t, h}} \geq C\left(\|\boldsymbol{w}\|_{t, h}+|\|r\||_{t, h}\right) \quad \forall(\boldsymbol{w}, r) \in \boldsymbol{V}_{h} \times Q_{h}
$$

The previous a priori estimates are now valid with $\left\|\boldsymbol{u}-\boldsymbol{u}_{h}\right\|_{t}$ replaced by $\left\|\boldsymbol{u}-\boldsymbol{u}_{h}\right\|_{t, h}$ on the left hand sides, and with $\|\boldsymbol{u}-\boldsymbol{v}\|_{t, h}$ replaced by $\square \boldsymbol{u}-\boldsymbol{v} \rrbracket_{t, h}$ on the right hand side, respectively. As before, for a smooth solution we obtain an $\mathcal{O}\left(h^{k}\right)$ convergence rate.

The modification needed for the a posteriori estimate is to add the term $t^{2} h_{K}^{-1}\left\|\boldsymbol{u}_{h}\right\|_{0, \partial K \cap \partial \Omega}^{2}$ to $E_{K}\left(\boldsymbol{u}_{h}, p_{h}\right)^{2}$.

\section{References}

[1] G. Allaire. Homogenization of the Navier-Stokes equations in open sets perforated with tiny holes. I. abstract framework, a volume distribution of holes. Arch. Rational Mech. Anal., 113(3):209-259, 1990.

[2] G. Allaire. Homogenization of the Navier-Stokes equations in open sets perforated with tiny holes. II. Noncritical sizes of the holes for a volume distribution and a surface distribution of holes. Arch. Rational Mech. Anal., 113(3):261-298, 1990.

[3] T. Arbogast and H. L. Lehr. Homogenization of a Darcy-Stokes system modeling vuggy porous media. Comput. Geosci., 10(3):291-302, 2006.

[4] D. N. Arnold, F. Brezzi, and M. Fortin. A stable finite element for the Stokes equations. Calcolo, 21(4):337-344 (1985), 1984.

[5] S. C. Brenner and L. R. Scott. The Mathematical Theory of Finite Element Methods, volume 15 of Texts in Applied Mathematics. SpringerVerlag, New York, second edition, 2002.

[6] P. Clément. Approximation of finite element functions using local regularization. RAIRO Num. Anal., 9:77-84, 1975.

[7] L.P. Franca and R. Stenberg. Error analysis of some Galerkin leastsquares methods for the elasticity equations. SIAM J. Num. Anal., 28(6):1680-1699, 1991. 
[8] P. Hansbo and M. Juntunen. Weakly imposed Dirichlet boundary conditions for the Brinkman model of porous media flow. Applied Numerical Mathematics, 59(6):1274-1289, 2009.

[9] M. Juntunen and R. Stenberg. Computations with finite element methods for the brinkman problem. Helsinki University of Technology Institute of Mathematics Research Report, A569, 2009.

[10] M. Juntunen and R Stenberg. A residual based a posteriori estimator for the reaction-diffusion problem. C. R. Math. Acad. Sci. Paris, 2009.

[11] T. Lévy. Loi de Darcy ou loi de Brinkman? C. R. Acad. Sci. Paris Sér. II Méc. Phys. Chim. Sci. Univers Sci. Terre, 292(12):871-874, Erratum (17):1239, 1981.

[12] K. A. Mardal, Xue-Cheng Tai, and R. Winther. A robust finite element method for Darcy-Stokes flow. SIAM J. Numer. Anal., 40(5):1605-1631 (electronic), 2002.

[13] J. Nitsche. Über ein Variationsprinzip zur Lösung von DirichletProblemen bei Verwendung von Teilräumen, die keinen Randbedingungen unterworfen sind. Abh. Math. Sem. Univ. Hamburg, 36:9-15, 1970/1971.

[14] J. Pitkäranta. Boundary subspaces for the finite element method with Lagrange multipliers. Numer. Math., 33:273-289, 1979.

[15] K. R. Rajagopal. On a hierarchy of approximate models for flows of incompressible fluids through porous solids. Math. Models Methods Appl. Sci., 17(2):215-252, 2007.

[16] R. Stenberg. On some techniques for approximating boundary conditions in the finite element method. J. Comput. Appl. Math., 63(1-3):139-148, 1995 .

[17] R. Verfürth. Error estimates for a mixed finite element approximation of the Stokes equations. RAIRO Anal. Numer., 18:175-182, 1984.

[18] R. Verfürth. A Review of a Posteriori Error Estimation and Adaptive Mesh-Refinement Techniques. Teubner Verlag and J. Wiley, Stuttgart, 1996. 

(continued from the back cover)

A562 Antti H. Niemi

A bilinear shell element based on a refined shallow shell model

December 2008

A561 Antti Hannukainen, Sergey Korotov, Michal Krizek

On nodal superconvergence in 3D by averaging piecewise linear, bilinear, and trilinear $\mathrm{FE}$ approximations

December 2008

A560 Sampsa Pursiainen

Computational methods in electromagnetic biomedical inverse problems

November 2008

A559 Sergey Korotov, Michal Krizek, Jakub Solc

On a discrete maximum principle for linear FE solutions of elliptic problems with a nondiagonal coefficient matrix

November 2008

A558 José Igor Morlanes, Antti Rasila, Tommi Sottinen

Empirical evidence on arbitrage by changing the stock exchange

December 2008

A556 Lourenço Beirão da Veiga, Jarkko Niiranen, Rolf Stenberg

A posteriori error analysis for the Morley plate element with general boundary conditions

December 2008

A555 Juho Könnö, Rolf Stenberg

Finite element analysis of composite plates with an application to the paper cockling problem

December 2008

A554 Lasse Leskelä

Stochastic relations of random variables and processes

October 2008

A553 Rolf Stenberg

A nonstandard mixed finite element family

September 2008 
HELSINKI UNIVERSITY OF TECHNOLOGY INSTITUTE OF MATHEMATICS RESEARCH REPORTS

The reports are available at $h t t p: / / m a t h . t k k . f i / r e p o r t s /$.

The list of reports is continued inside the back cover.

A567 Antti Hannukainen, Sergey Korotov, Michal Krizek

On a bisection algorithm that produces conforming locally refined simplicial meshes

April 2009

A566 Mika Juntunen, Rolf Stenberg

A residual based a posteriori estimator for the reaction-diffusion problem

February 2009

A565 Ehsan Azmoodeh, Yulia Mishura, Esko Valkeila

On hedging European options in geometric fractional Brownian motion market model

February 2009

A564 Antti H. Niemi

Best bilinear shell element: flat, twisted or curved?

February 2009

A563 Dmitri Kuzmin, Sergey Korotov

Goal-oriented a posteriori error estimates for transport problems

February 2009

ISBN 978-951-22-9604-0 (print)

ISBN 978-951-22-9605-7 (PDF)

ISSN 0784-3143 (print)

ISSN 1797-5867 (PDF) 